\title{
Allgemeine und abgekürzt zitierte Literatur, Lehrbücher, Kommentare und Einführungen (Literatur vor 1978 s 8. Aufl)
}

\author{
Ahrens \\ Der Wettbewerbsprozess ${ }^{7}, 2013$, bearbeitet von Achilles, Ahrens, Bacher, Bähr, \\ Ammendola \\ Ann/Barona \\ Bartenbach/Volz \\ Bartenbach/Volz VergRl \\ Baumbach/Hefermehl \\ Bausch \\ Beier/Haertel/Schricker \\ (Hrsg) \\ Beil \\ Benkard \\ Benkard-EPÜ \\ Bertschinger/Geiser/ \\ Münch \\ Blumer \\ Boemke/Kursawe \\ Boeters \\ Brändel \\ Braitmayer/van Hees \\ Bremi \\ Bruchhausen \\ Buddeberg \\ Bühring \\ Büscher/Dittmer/ \\ Schiwy (Hrsg) \\ Bunte (Hrsg) \\ Busche/Stoll/Wiebe \\ Cepl/Voß \\ Chrocziel \\ Cohausz \\ Bornkamm, Büttner, Jestaedt, Scharen, Singer, Spätgens \\ La brevettabilità nella Convenzione di Monaco, Mailand 1981 \\ Schuldrechtsmodernisierung und gewerblicher Rechtsschutz, 2002 \\ Arbeitnehmererfindungsgesetz ${ }^{5}, 2013$ \\ Arbeitnehmererfindervergütung ${ }^{4}, 2017$ \\ Warenzeichenrecht ${ }^{12}, 1985$ \\ Nichtigkeitsrechtsprechung in Patentsachen, Bd 1 (BGH 1994-1998), Bd 2 \\ (BPatG 1994-1998), 2000, Bd 3 (BGH 1999-2001), 2002; Bd 4 \\ (BGH 1986-1993), 2007 \\ Europäisches Patentübereinkommen, Münchner Gemeinschaftskommentar \\ (zit MGK/Bearbeiter), 2014 bis 30. Lieferung \\ Grundzüge des gewerblichen Rechtsschutzes und des Erfinderrechts, 1961 \\ Patentgesetz Gebrauchsmustergesetz ${ }^{11}$, 2015, bearbeitet von Asendorf, Bacher, \\ Deichfuß, Engel, Fricke, Goebel, Grabinski, Hall, Kober-Dehm, Melullis, Nobbe, \\ Rogge, Schäfers, Scharen, Schmidt, Schramm, Schwarz, Tochtermann, Ullmann. \\ Zülch \\ Europäisches Patentübereinkommen ${ }^{3}$, 2019, bearbeitet von Adam, Bacchin, \\ Birken, Ehlers, Fritsche, Grabinski, Henke, Irmscher, Joos, Keussen, Kinkeldey, \\ Kley, Kolle, Melullis, Osterrieth, Quarch, Scharen, Söldenwagner, Thums, \\ Unland, Wieser \\ Schweizerisches und europäisches Patentrecht, 2002, bearbeitet von \\ Bertschinger, Blumer, Comte ua \\ Formulierung und Änderung der Patentansprüche im europäischen Patent- \\ recht, 1998 \\ Gesetz über Arbeitnehmererfindungen, 2015, bearbeitet von Boemke, Enge- \\ mann, Gennen, Hoppe-Jänich, Kursawe, Nebel, Raif, Sachadae, Ulrici \\ Handbuch Chemiepatent ${ }^{2}, 1989$ \\ Technische Schutzrechte, 1995 \\ Verfahrensrecht in Patentsachen ${ }^{4}, 2010$, 5. Aufl angekündigt \\ The European Patent Convention, 2008 \\ Patent-, Sortenschutz- und Gebrauchsmusterrecht, 1985 \\ Beck'sche Formularsammlung zum gewerblichen Rechtsschutz mit Urheber- \\ recht $^{3}, 2015$ \\ Gebrauchsmustergesetz ${ }^{8}$, 2011 \\ Gewerblicher Rechtsschutz, Urheberrecht, Medienrecht ${ }^{3}$, 2015, PatG und \\ GebrMG bearbeitet von Obenland, von Samson-Himmelstjerna, Lippich, von \\ Pichler, Trimborn, Köhler, Kanz, Lunze, Kasper, Schoenen (4. Aufl angekündigt \\ für Herbst 2020) \\ Lexikon des Rechts, Wettbewerbsrecht, Gewerblicher Rechtsschutz, 1996 \\ TRIPs, Internationales und europäisches Recht des geistigen Eigentums ${ }^{2}$, \\ 2013 \\ Prozesskommentar zum Gewerblichen Rechtsschutz², 2018, bearbeitet von \\ Augenstein, Bacher, Cassardt, Cepl, Guhn, Haft, Hahn, Jacobs, Lunze, Matthes, \\ Müller, Nielen, Rinken, Rüting, Schilling, Thomas, Tochtermann, Voß, Werner, \\ Zigann, Zöllner \\ Einführung in den Gewerblichen Rechtsschutz und das Urheberrecht ${ }^{2}, 2002$ \\ Patente und Muster, 1993
}


Cole (Hrsg)

Däbritz/Jesse/Bröcher

Dybdahl-Müller

Eichmann/v. Falcken-

stein/Kühne

Eisenmann/Jautz

Ekey/Bender/

Fuchs-Wissemann

Engels

Ensthaler

Erdmann/Rojahn/Sosnitza

Fezer

Fezer

Fischer

Fitzner/Lutz/Bodewig

Formular-Kommentar

Gall

Gleiss

Geissler

Goebel

Götting

Götting/Hetmank/

Schwipps

Götting/Meyer/

Vormbrock (Hrsg)

Gruber

Gruber/von Zumbusch/

Haberl/Oldekop

Günther/Beyerlein

Haedicke

Haedicke/Timmann

Haedicke

Hassemer

Heinrich

Hellebrandt/Rabe

Hilti

Hirsch

Hirsch/Hansen

Hoeren

Hüttermann

Ingerl/Rohnke

Jestaedt

Keukenschrijver
C.I.P.A. Guide to the Patent Acts ${ }^{7}, 2012$

Patente. Wie versteht man sie? Wie bekommt man sie? Wie geht man mit ihnen um? 2009

Europäisches Patentrecht ${ }^{3}, 2009$

Designgesetz ${ }^{5}, 2015$

Grundriss Gewerblicher Rechtsschutz und Urheberrecht ${ }^{10}, 2015$

Markenrecht ${ }^{4}, 2019$, bearbeitet von Bender, Dück, Eisfeld ua

Patent-, Marken- und Urheberrecht ${ }^{9}, 2015$ (bis 7. Auflage: Ilzhöfer)

Gewerblicher Rechtsschutz und Urheberrecht ${ }^{3}, 2009$

Handbuch des Fachanwalts Gewerblicher Rechtsschutz ${ }^{3}, 2018$

Markenrecht ${ }^{5}, 2019$

Handbuch der Markenpraxis ${ }^{3}, 2016$

Grundzüge des gewerblichen Rechtsschutzes², 1986

Patentrechtskommentar ${ }^{4}$, 2012, bearbeitet von Fitzner, Lutz, Bodewig (zugl Hrsg), Ahrens, Beckmann, Einsele, Eisenrauch, Ensthaler, Feuerlein, Fischer, Gleiter, Haertel, Hauck, Heinrich, Hofmeister, Hössle, Kiefer, Kircher, Kubis, Loth, Metternich, Müller, Münch, Pitz, Rauch, Rinken, Schnekenbühl, Stortnik, Voß, Wickenhöfer, Wilhelmi

Bd 3, Handels- und Wirtschaftsrecht III, Gewerblicher Rechtsschutz ${ }^{21}, 1979$

Die europäische Patentanmeldung und der PCT in Frage und Antwort ${ }^{7}, 2006$

Gewerblicher Rechtsschutz, 2002

Halbleiterschutzgesetz/Semiconductor Protection Act, Textausgabe mit

Erläuterungen, 1988

Das Patentgesetz in der Praxis des Deutschen Patentamts, 1984

Gewerblicher Rechtsschutz ${ }^{10}$, 2014, begründet von Hubmann

Patentrecht, 2014

Gewerblicher Rechtsschutz und Wettbewerbsrecht ${ }^{2}, 2020$

Gewerblicher Rechtsschutz und Urheberrecht ${ }^{7}, 2015$

Europäisches und internationales Patentrecht ${ }^{7}, 2012$

DesignG, 2015

Patentrecht ${ }^{4}, 2018$

Handbuch des Patentrechts ${ }^{2}, 2020$ (auch englisch: Patent Law, 2014), bearbeitet von Bühler, Bukow, Chakraborty, Haedicke, Harbsmeier, Hartmann, Kamlah, Landry, Nack, Nägerl, Nestler, Pansch, Schnekenbühl, Stief, Timmann, Verhauwen, Wollenschlaeger, Zigann

Patente und Piraten, 2011

Patentecht mit Arbeitnehmererfindungsrecht, Gebrauchsmusterrecht, Sortenschutzrecht und Patentmanagement ${ }^{2}, 2015$

PatG/EPÜ2, 2010 (Schweiz)

Lizenzsätze für technische Erfindungen ${ }^{5}, 2017$

Europäisches und schweizerisches Patent- und Patentprozessrecht, 2008

Chemie-Erfindungen und ihr Schutz nach neueren Gerichtsentscheidungen und Entscheidungen der Beschwerdekammern des Europäischen Patentamts $^{2}, 1987$

Der Schutz von Chemie-Erfindungen, 1995

Der Schutz von Mikrochips in der Bundesrepublik Deutschland, 1988

Einheitspatent und Einheitliches Patentgericht, 2017

Markengeset $z^{3}, 2010$

Patentrecht ${ }^{2}, 2008$

Sortenschutzrecht ${ }^{2}, 2017$ (Keukenschrijver SortSch) 


\author{
Keukenschrijver \\ Kley/Gundlach \\ Köhler/Bornkamm \\ Kohler \\ Kraßer \\ Kraßer/Ann
}

Kremnitz

Kur/v. Bomhard/Albrecht

Kucsko

Kühnen

Kühnen Hdb

Lehr/Göckmann

Lendvai/Rebel

Liedel

Loth

Maksymiw

Mathély

Melullis

Mes

Metzger/Zech

MGK

Müller-Guggenberger/

Bieneck (Hrsg)

Münch

Münchner Anwaltshand-

buch Gewerblicher

Rechtsschutz

Münchner Prozessformu-

larbuch Gewerblicher

Rechtsschutz, Urheber-

recht, Presserecht

Nirk

Nirk/Ullmann/Metzger

Osterrieth

Pahlow

Paterson

Paul

Pfaff/Osterrieth

Pierson/Ahrens/Fischer

Rehmann

Reichel

Reimer/Schade/Schippel

Ring

Ritscher/Holzer/Kasche

Scheer

Scheer
Patentnichtigkeitsverfahren ${ }^{6}$, 2016, Neuauflage für Dezember 2020 angekün-

digt

Kommentar zum EPÜ 2000, Loseblatt, 2008

Wettbewerbsrecht ${ }^{38}, 2020$ (jetzt: Köhler/Bornkamm/Feddersen)

Lehrbuch des Patentrechts, 1908

Patentrecht, WissFS 50 Jahre BGH Bd 2, 647

Patentrecht ${ }^{7}, 2016$ (bis 4. Aufl 1986 Bernhardt/Kraßer Lehrbuch des Patentrechts, 5. und 6. Aufl Kraßer Patentrecht, 8. Aufl unter der alleinigen Bezeichnung Ann angekündigt)

Patentrecht von A-Z (1976/84)

Markenrecht ${ }^{2}, 2019$

Geistiges Eigentum, 2003

Die Teilung des Patents, 2000 (Kühnen Teilung)

Handbuch der Patentverletzung ${ }^{12}$, 2020 (bis 4. Aufl Kühnen/Geschke Die

Durchsetzung von Patenten in der Praxis), auch engl Ausgabe unter dem

Titel: Patent Litigation Proceedings in Germany, 2013

Leitfaden zum gewerblichen Rechtsschutz, Urheberrecht und Multimedia, 1999

Gewerbliche Schutzrechte ${ }^{7}, 2017$

Das deutsche Patentnichtigkeitsverfahren, 1979

Gebrauchsmustergesetz ${ }^{2}$, 2017, bearbeitet von Loth, Hauck, Pantze, Stock

Schutz technischer Erfindungen, 2017

Le Droit Européen des Brevets d'Invention, Paris 1978

Patentrecht ${ }^{2}, 2013$

Patentgesetz und Gebrauchsmustergesetz ${ }^{5}, 2020$

Sortenschutzrecht, 2016

Münchener Gemeinschaftskommentar zum EPÜ, 2014 bis 30. Lieferung

Wirtschaftsstrafrecht ${ }^{6}, 2014$

Patentbegriffe von A bis Z, 1992

hrsg von Hasselblatt, 5. Aufl 2017

hrsg von Mes, 5. Aufl 2018

Gewerblicher Rechtsschutz, 1981

Patentrecht. Mit Gebrauchsmuster- und Sortenschutzrecht ${ }^{4}, 2018$

Patentrecht, Einführung für Studium und Praxis ${ }^{5}, 2015$

Patentvertragsrecht, 2017

The European Patent System, 1992

Lexikon Gewerblicher Rechtsschutz, angekündigt für Oktober 2019

Lizenzverträge, Formularkommentar ${ }^{4}, 2018$

Recht des geistigen Eigentums, 2014

Designrecht ${ }^{2}, 2014$

Gebrauchsmuster- und Patentecht - praxisnah $^{6}, 2003$

Das Recht der Arbeitnehmererfindung ${ }^{8}$, 2007, bearbeitet von Himmelmann, Leuze, Rother und Trimborn

Gewerblicher Rechtsschutz, 2019

Patentrecht, Entwicklungen 2007

Deutsches Patent-, Gebrauchsmuster, Warenzeichen-, Wettbewerbs- und Arbeitnehmererfindungs-Recht ${ }^{41}, 1997$

Die Internationale PCT-Anmeldung. Das Europäische Patent. Das Gemeinschaftspatent ${ }^{9}, 1996 / 97$ 
Schennen

Schramm PVP

Schulte

Schweizer/Zech

Singer

Singer/Stauder/Luginbühl

Stadler/Koller (Hrsg)

Stammberger/Lippelt

Straus

Steckler

Stockmair

Ströbele/Hacker/Thiering

Teplitzky

V. Tetzner

Thouvenin

Tilmann

Tilmann/Plassmann

Troller

van Venrooy

Véron/Cottier

Volmer/Gaul

von Büren/Marbach/

Ducrey

von Hellfeld

von Schultz

Weiser

Werum

Wiltschek

WIPO (Hrsg)

Witte/Vollrath

Wuesthoff/

Leßmann/Würtenberger
Die Verlängerung der Patentlaufzeit für Arzneimittel im Gemeinsamen Markt, 1993

Der Patentverletzungsprozess ${ }^{7}$, 2013, bearbeitet von Kilchert, Kaess, Schneider, Oldekop, Donle, Ebner

Patentgesetz mit EPÜ ${ }^{10}$, 2017, bearbeitet von Schulte, Moufang, Püschel, Rin-

ken, Rudloff-Schäffer, Schell, Schulte, Voit, Voß

Patentgesetz PatG, 2016 (Schweiz)

Das neue europäische Patentsystem, 1979

Europäisches Patentübereinkommen ${ }^{8}$, 2019; weitere Bearbeiter: Almer,

Blumer, Bostedt, Bremi, Brückner, Bühler, Haugg, Hesper, Klopschinski,

Kroher, Kunz-Hallstein, Lindner, Luginbühl, Mathoi, Neumann, Plouard, Podbielski, Preller, Quarch, Schauwecker, Schmitz, Stauder, Steinbrener, Teschemacher, Weiss, Zimmermann

Patentgesetz. GMG - PatV-EG. Kommentar, bearbeitet von Adocker, Alge, Anderl, 2019

Deutsches und Europäisches Patentrecht ${ }^{3}, 2013$

The present state of the patent system in the European Union as compared with the situation in the United States and Japan, 1997

Grundzüge des IT-Rechts ${ }^{3}, 2011$

The Protection of Technical Innovations and Designs in Germany, 1994

Markengesetz ${ }^{12}$, 2018, bearbeitet von Hacker, Knoll, Miosga, Ströbele, Thiering

Wettbewerbsrechtliche Ansprüche und Verfahren ${ }^{11}, 2016$ (Hrsg Bacher)

Leitfaden des Patent-, Gebrauchsmuster- und Arbeitnehmererfindungsrechts der Bundesrepublik Deutschland ${ }^{3}, 1983$

Funktionale Systematisierung von Wettbewerbsrecht (UWG) und Immaterialgüterrechten, Diss Zürich 2007

Neue Überlegungen im Patentrecht, GRUR 2005, 904; GRUR 2006, 824; GRUR 2008, 312

Einheitspatent, Einheitliches Patentgericht, angekündigt für Herbst 2018

Immaterialgüterrecht ${ }^{3}$, 2 Bde, 1983/85

Patentrecht einschließlich Arbeitnehmererfindungsrecht und Vertragsrecht, 1996

Concise International and European IP Law - TRIPS, Paris Convention, European Enforcement and Transfer of Technology ${ }^{3}, 2014$

Arbeitnehmererfindungsgesetz², 1983

Immaterialgüter- und Wettbewerbsrecht, 2008

Verfahrensrecht im gewerblichen Rechtsschutz, 2015

Kommentar zum Markenrecht ${ }^{3}, 2012$

Patentgesetz, Gebrauchsmustergesetz [Österreich] $]^{3}, 2016$

Der Schutz von Halbleitererzeugnissen der Mikroelektronik im deutschen

Rechtssystem, 1990

Patentrecht, Wien 2006

Introduction to Intellectual Property Theory and Practice, 1997

Praxis der Patent- und Gebrauchsmusteranmeldung ${ }^{6}, 2008$

Handbuch zum deutschen und europäischen Sortenschutzrecht, 2 Bde, 1999,

2. Aufl unter der Bezeichnung Leßmann/Würtenberger Deutsches und europäisches Sortenschutzrecht 2009 


\section{Festschriften und Sammelwerke}

5. St. Galler Internationales

Immaterialgüterrechts-

forum 2001

Album Amicorum

voor Sonja Boekman, 16 januari 1997

EPOscript 1

Festgabe zum

Schweiz. Juristentag 1994

Festgabe Schweiz. Juristenverein 2000

FS AIPPI 1897-1997 (1997)

FS H.-J. Ahrens (2016)

FS W. Ballhaus

FS K. Bartenbach (2005)

FS F.-K. Beier (1996)

FS F.-K. Beier (1996)

FS K. Bepler (2012)

FS V. Beuthien

FS U. Blaurock (2013)

FS Blum (1978)

FS R. Blum (1989)

FS K.-H. Böckstiegel (2001)

$$
\text { Li }
$$
Direktor des Instituts für Luft- und Weltraumrecht und des von ihm gegründeten Lehrstuhls für Internationales Wirtschaftsrecht, hrsg von R. Briner u.a., 2001

FS K.-H. Böckstiegel (2001) Luft- und Weltraumrecht im 21. Jahrhundert, liber amicorum Karl-Heinz Böckstiegel, anlässlich seines Ausscheidens ... sowie zum 50-jährigen Bestehen der von ihm herausgegebenen Zeitschrift für Luft- und Weltraumrecht, hrsg von M. Benkö ua, 2001

FS M.M. Boguslavskij (2004)

FS J. Bornkamm (2014)

FS H.E. Brandner (1996)

Neueste Entwicklungen im europäischen und internationalen Immaterialgüterrecht, hrsg von C. Baudenbacher ua (2002)

$=$ BIE 1997, $1 \mathrm{ff}$ Cookson, Nowak, Thierbach (Hrsg) Genetic Engineering - The New Challenge, 1993

Aspekte des Wirtschaftsrechts, hrsg von H.U. Walder, T. Jaag und D. Zobl (1994)

Recht und Internationalisierung, Festgabe gewidmet dem Schweizerischen Juristenverein anlässlich des Juristentags 2000 in St. Gallen ..., hrsg von Ch. Meier-Schatz und R. Schweizer, 2000

AIPPI und die Entwicklung des gewerblichen Rechtsschutzes 1897-1997, Ausgabe zur Hundertjahrfeier, hrsg von S. Amberg, 1997

Rechtsdurchsetzung - Rechtsverwirklichung durch materielles Recht und Verfahrensrecht, Festschrift für Hans-Jürgen Ahrens zum 70. Geburtstag, hrsg von Büscher/Erdmann/Fuchs, 2016

= GRUR 1985, 575ff

Festschrift für Kurt Bartenbach zum 65. Geburtstag, hrsg von M. Haesemann, K. Gennen und B. Bartenbach, 2005

= GRUR Int 1996, 273

= Aktuelle Herausforderungen des geistigen Eigentums, Festgabe für Friedrich-Karl Beier zum 70. Geburtstag, hrsg von J. Straus, 1996 Arbeitsgerichtsbarkeit und Wissenschaft, Festschrift für Klaus Bepler zum 65. Geburtstag, hrsg von G. Thüsing, M. Creutzfeldt, P. Hanau, H. Wißmann Von der Sache zum Recht, Festschrift für Volker Beuthien zum 75. Geburtstag, hrsg von M. Schöpflin, F. Meik, H.-O. Weber und J. Bandte, 2009

Einheit und Vielheit im Unternehmensrecht, Festschrift für Uwe Blaurock zum 70. Geburtstag, hrsg von P. Jung, Ph. Lamprcht, K. Blasek und M. Schmidt-Kessel, 2013

Beiträge zu Fragen des gewerblichen Rechtsschutzes, Festschrift Blum, 1978 Festschrift für Dr. Rudolf E. Blum, 1989

aw of international business and dispute settlement in the 21st century, Liber amicorum Karl-Heinz Böckstiegel anlässlich seines Ausscheidens als Russland im Kontext der internationalen Entwicklung, Festschrift für Mark Moiseevič Boguslavskij, hrsg von A. Trunk, R. Knieper und A.G. Svetlanov, 2004 Festschrift für Joachim Bornkamm zum 65. Geburtstag, hrsg von W. Büscher, W. Erdmann, M. Haedicke, H. Köhler und M. Loschelder, 2014

Festschrift für Hans Erich Brandner zum 70. Geburtstag, hrsg von G. Pfeiffer, J. Kummer, S. Scheuch, 1996 
FS A. Braun

FS K. Bruchhausen

FS W. Büscher (2018)

FS D. Coester-Waltjen (2015) Zwischenbilanz, Festschrift für Dagmar Coester-Waltjen, hrsg von K. Hilbig-

FS Constantinesco (1983)

FS L. David (1996)

FS A. Deringer (1993)

FS E. Deutsch (1999)

FS A. Dietz (2001)

FS K. Doehring (1989)

FS U. Doepner (2008)

FS W. Döser (1999)

FS J. Druey (2002)

FS G. Eisenführ (2003)

FS U. Eisenhardt (2007)

FS W. Erdmann (2002)

FS U. Everling (1995)

FS K.-H. Fezer (2016)

FS W. Fikentscher (1998)

FS A.-C. Gaedertz (1992)

FS D. Gaul (1980)

FS D. Gaul (1992)

FS K. Geiß (2000)

FS H.-D. Gesthuysen (2010)

FS I. Griss (2011)

FS H. Hahn (1997)

FS H. Hattenhauer (2003)

FS M. Hecker (2017)

FS W. Hefermehl (1996)

FS A. Heldrich (2005)

Jura vigilantibus, Antoine Braun, les droits intellectuels, le barreau, hrsg von F. de Visscher, Brüssel 1994

$=$ GRUR 1993, $163 \mathrm{ff}$

H.-J. Ahrens/J. Bornkamm/K.-H. Fezer/Th. Koch/M.-R. McGuire/G. Würtenberger (Hrsg): Festschrift für Wolfgang Büscher, 2018

Lugeni, D. Jakob, G. Mäsch, Ph. Reuß, Ch. Schmid, 2015

Rechtsvergleichung, Europarecht und Staatenintegration, 1983

Binsenweisheiten des Immaterialgüterrechts, Festschrift für Lucas David zum 60. Geburtstag, hrsg von M. Kurer ua, Zürich 1996

Europarecht, Kartellrecht, Wirtschaftsrecht, Festschrift für Arved Deringer, hrsg von U. Everling, K.-H. Narjes, J. Sedemund, 1993

Festschrift für Erwin Deutsch zum 70. Geburtstag, hrsg von H.-J. Ahrens ua, 1993

Urheberrecht - Gestern - Heute - Morgen, Festschrift für Adolf Dietz zum 65. Geburtstag, hrsg von P. Ganea, Ch. Heath und G. Schricker, 2001

Staat und Völkerrechtsordnung, Festschrift für Karl Doehring, hrsg von

K. Hailbronner, G. Ress und T. Stein, 1989

Festschrift für Ulf Doepner zum 65. Geburtstag, hrsg von U. Reese, F.-E. Hufnagel und A. Lensing-Kramer, 2008

The international lawyer, Freundesgabe für Wulf H. Döser, hrsg von F. Kübler ua, 1999

Festschrift für Jean Nicolas Druey zum 65. Geburtstag, hrsg von R. Schweizer, H. Burkert, V. Gasser, 2002

Festschrift für Günther Eisenführ zum 70. Geburtstag, hrsg von Ch. Spintig und J. Ehlers, 2003

Festschrift für Ulrich Eisenhardt zum 70. Geburtstag, hrsg von U. Wackerbarth, Th. Vormbaum und H. P. Marutschke, 2007

Festschrift für Willi Erdmann [zum 65. Geburtstag], hrsg von H.-J. Ahrens, J. Bornkamm, W. Gloy, J. Starck, J. v. Ungern-Sternberg, 2002

Festschrift für Ulrich Everling, hrsg von O. Due, M. Lutter, J. Schwarze, 1995

Marktkommunikation zwischen Geistigem Eigentum und Verbraucherschutz, Festschrift für Karl-Heinz Fezer zum 70. Geburtstag, hrsg von W. Büscher, J. Glöckner, A. Nordemann, Ch. Osterrieth, R. Rengier, 2016

Festschrift für Wolfgang Fikentscher zum 70. Geburtstag, hrsg von B. Großfeld, R. Sack, Th. Möllers, J. Drexl und A. Heinemann, 1998

Festschrift für Alfred-Carl Gaedertz zum 70. Geburtstag, hrsg von G. Wild, I.M. Schulte-Franzheim und M. Lorenz-Wolf, 1992

Festschrift für Dieter Gaul zum fünfundzwanzigjährigen Dozentenjubiläum, herausgegeben von der Technischen Akademie Wuppertal, 1980

Festschrift für Dieter Gaul zum 70. Geburtstag, hrsg von D. Boewer, B. Gaul, 1992

Festschrift für Karlmann Geiß zum 65. Geburtstag, hrsg von H.-E. Brandner, H. Hagen, R. Stürner, 2000

$=$ Mitt 2010, $145 \mathrm{ff}$

Festschrift für Irmgard Griss, hrsg von B. Schenk, E. Lovrek, G. Musger, M. Neumayr, 2011

Währung und Wirtschaft. Das Geld im Recht, FS für Prof. Dr. Hugo J. Hahn zum 70. Geburtstag, hrsg von A. Weber ua, 1997

Der praktische Nutzen der Rechtsgeschichte, Hans Hattenhauer zum

8. September 2001, hrsg von J. Eckert, 2003

Liber Amicorum für Manfred Hecker, hrsg von M. Ruttig und I. Jung, 2017

$=$ GRUR 1996, 515

Festschrift für Andreas Heldrich zum 70. Geburtstag, hrsg von St. Lorenz ua, 2005 
FS H. Helm (2002)

FS H. Helmrich (1994)

FS P. Hertin (2000)

FS G. Hirsch (2008)

FS M. Hoffmann-Becking (2013)

FS J. Hruschka (2005)

FS H. Hubmann (1985)

FS U. Immenga (2004)

FS R. Jacobs (2011)

FS O. Kimminich (1999)

FS H.-P. Kirchhof (2003)

FS H. Kirchner (1985)

FS T. Kigawa (2005)

FS Z. Kitagawa (1992)

Festgabe M. Kisseler

FS R. Klaka (1987)

FS H. Köhler (2015)

FS R. König (2003)

FS Kolle/Stauder (2005)

FS H.-G. Koppensteiner (2001)

FS A. Kraft (1998)

FS A. Krämer (2009)

FS R. Kraßer (2004)

FS H. Krüger (2001)

FS G. Krüger-Nieland

FS W. Küttner (2006)

FSD. Leipold

FS M. Levin (2008)

FS O. Lieberknecht (1997)
Recht und Wettbewerb, Festschrift für Horst Helm zum 65. Geburtstag, hrsg von Th. Bopp und W. Tilmann, 2002

Festschrift für Herbert Helmrich zum 60. Geburtstag, hrsg von K. Letzgus, H. Hill, H.H. Klein, D. Kleinert, G.-B. Oschatz, H. de With, 1994

Festschrift für Paul W. Hertin zum 60. Geburtstag, hrsg von Ch. Scherz und H.-J. Omsels, 2000

Festschrift für Günter Hirsch zum 65. Geburtstag, hrsg von G. Müller, E. Osterloh, T. Stein, 2008

Festschrift für Michael Hoffmann-Becking, hrsg von G. Krieger, M. Lutter und K. Schmidt, 2013

Philosophia Practica Universalis, Festschrift für Joachim Hruschka zum

70. Geburtstag, hrsg von B. Sharon Byrd ua, 2005

Beiträge zum Schutz der Persönlichkeit und ihrer schöpferischen Leistungen,

Festschrift für Heinrich Hubmann zum 70. Geburtstag, hrsg von H. Forkel und A. Kraft, 1985

Wirtschafts- und Privatrecht im Spannungsfeld von Privatautonomie, Wettbewerb und Regulierung, Festschrift für Ulrich Immenga zum 70. Geburtstag, hrsg von A. Fuchs, H.-P. Schwintowski und Daniel Zimmer, 2004

= GRUR 2011, 1057

Vielfalt des Wissenschaftsrechts, Gedächtnisschrift für Prof. Dr. Otto

Kimminich, hrsg von Ch. Fläming ua, 1999

Insolvenzrecht im Wandel der Zeit, Festschrift für Hans-Peter Kirchhof zum

65. Geburtstag, hrsg von W. Gerhard ua, 2003

Festschrift für Hildebert Kirchner zum 65. Geburtstag, hrsg von W. Dietz und

D. Pannier, 1985

Ach; so ist das! Liber amicorum Prof. Dr. Toichiro Kigawa zum 80. Geburtstag, hrsg v. J. Westhoff ua, 2005

Wege zum japanischen Recht. Festschrift für Zentaro Kitagawa zum

60.Geburtstag am 5.4.1992, hrsg von H.G. Leser und T. Isomura, 1992

$=\mathrm{WRP} 1997,887 \mathrm{ff}$

Festschrift für Rainer Klaka, hrsg von G. Herbst, 1987

Festschrift für Helmut Köhler zum 70. Geburtstag, hrsg von Chr. Alexander, J. Bornkamm, B. Buchner, J. Fritzsche und T. Lettl, 2015

Materielles Patentrecht, Festschrift für Reimar König, hrsg von Ch. Ann, W. Anders, U. Dreiss, B. Jestaedt, D. Stauder, 2003

„.... und sie bewegt sich doch!“ - Patent Law on the Move, Festschrift für Gert Kolle und Dieter Stauder, hrsg von A. Kur, St. Luginbühl, E. Waage (2005)

Beiträge zum Unternehmensrecht, Festschrift für Hans-Georg Koppensteiner zum 65. Geburtstag, hrsg. von E.A. Kramer ua, 2001

Festschrift für Alfons Kraft zum 70. Geburtstag, hrsg von G. Hönn ua, 1998

Festschrift für Achim Krämer zum 70. Geburtstag am 19. September 2009, hrsg von U. Blaurock, J. Bornkamm und Chr. Kirchberg, 2009

$=$ GRUR Int 2004, $695 \mathrm{ff}$

Wissenschaftsrecht im Umbruch, Gedächtnisschrift für Hartmut Krüger, hrsg von P. Hanau ua, 2001

$=$ GRUR 1980, $511 \mathrm{ff}$

Personalrecht im Wandel, Festschrift für Wolfdieter Küttner zum

70. Geburtstag, hrsg von P. Hanau, J. Röller, L. Macher und R. Schlegel, 2006

Festschrift für Dieter Leipold zum 70. Geburtstag, hrsg von R. Stürnerua, 2009

Festskrift till Marianne Levin, hrsg von A. Bakardjieva Engelbrekt, U. Bernitz, B. Domeij, A. Kur, P. J. Nordell (2008)

Festschrift für Otfried Lieberknecht zum 70. Geburtstag, hrsg von E. Niederleithinger ua, 1997 
FS W. Lindacher (2007)

FS. U. Loewenheim (2009)

FS W. Lorenz (1991)

FS M. Loschelder (2010)

FS R. Lukes (1990)

FS K.P. Mailänder (2006)

FS J. Mantscheff (2000)

FS R. Maunz (1981)

FS. K.-J. Melullis (2009)

FS F. Merz (1992)

FS P. Mes (2009)

FS P. Mes (2014)

FS E.-J. Mestmäcker (1996)

FS A. v. Mühlendahl (2005)

FS W.-D. Müller-Jahncke (2009)

FS H. Nagel (1987)

FS R. Nirk (1992)

FS W. Nordemann (1999)

FS W. Nordemann (2004)

FS W. Odersky (1996)

FS W. Oppenhoff (1980)

FS W. Oppenhoff (1985)

FS J. Pagenberg (2006)

FS G. Pfeiffer (1988)

FS H. Piper (1996)

FS A. Preu (1988)

FS K. Quack (1991)

FS G. Rahn (2011)
Facetten des Verfahrensrechts, liber amicorum Walter F. Lindacher zum 70. Geburtstag am 20. Februar 2007, hrsg v. W. Hau ua, 2007

Schutz von Kreativität und Wettbewerb, Festschrift für Ulrich Loewenheim zum 75. Geburtstag, hrsg v. R. Hilty, J. Drexl und W. Nordemann, 2009

Festschrift für Werner Lorenz zum siebzigsten Geburtstag, hrsg von B. Pfister und M.R. Will, 1991

Festschrift für Michael Loschelder, hrsg von W. Erdmann, M. Leistner, W. Rüffer, Th. Schulte-Beckhausen, 2010

Festschrift für Rudolf Lukes zum 65. Geburtstag, hrsg von H. Leßmann, B. Großfeld und L. Vollmer, 1990

Festschrift für Karl Peter Mailänder zum 70. Geburtstag, hrsg von K. Geiss, K. Gerstenmaier, R. Winkler, P. Mailänder, 2006

Technik und Recht, Festschrift für Jack Mantscheff zum 70. Geburtstag, hrsg von I. Jagenburg, 2000

Festschrift für Theodor Maunz zum 80. Geburtstag am 1. September 1981, hrsg von P. Lerche, H. Zacher und P. Badura, 1981

$=$ GRUR 2009, 193

Festschrift für Franz Merz zum 65. Geburtstag am 3. Februar 1992, hrsg von W. Gerhardt, W. Henckel, J. Kilger, G. Kreft, 1992

Festschrift für Peter Mes zum 65. Geburtstag, hrsg von G. Rother, M. Bergermann und A. Verhauwen, 2009

$=$ GRUR 2014, 1

Festschrift für Ernst-Joachim Mestmäcker zum siebzigsten Geburtstag, hrsg von U. Immenga ua, 1996

Harmonisierung des Markenrechts, Festschrift für Alexander von Mühlendahl, hrsg von V. von Bomhard, J. Pagenberg, D. Schennen, 2005

Pharmazie in Geschichte und Gegenwart, Festgabe für Wolf-Dieter MüllerJahncke zum 65. Geburtstag, hrsg von C.F. Telle und J. Telle, 2009

Beiträge zum internationalen Verfahrensrecht und zur Schiedsgerichtsbarkeit, Festschrift für Heinrich Nagel zum 75. Geburtstag, hrsg von W.J. Habscheid und K.H. Schwab, 1987

Festschrift für Rudolf Nirk zum 70. Geburtstag, hrsg von K. Bruchhausen, W. Hefermehl, P. Hommelhoff und H. Messer, 1992

Festschrift für Wilhelm Nordemann, hrsg von B. Zollner und U. Fitzner, 1999

Urheberrecht im Informationszeitalter, Festschrift für Wilhelm Nordemann zum 70. Geburtstag, hrsg von U. Loewenheim, 2004

Festschrift für Walter Odersky zum 65. Geburtstag am 17. Juli 1996, hrsg von

R. Böttcher, G. Hueck und B. Jähnke, 1996

$=$ GRUR 1980, $333 \mathrm{ff}$

Festschrift für Walter Oppenhoff zum 80. Geburtstag, hrsg von W.Jagenburg, G. Meier-Reimer, T. Verhoeven, 1985

Festschrift für Jochen Pagenberg zum 65. Geburtstag, hrsg von D. Beier, L. Brüning-Petit und Ch. Heath, 2006

Strafrecht, Unternehmensrecht, Anwaltsrecht, Feststchrift für Gerd Pfeiffer zum Abschied aus dem Amt als Präsident des Bundesgerichtshofes, hrsg von O.F. Freiherr von Gamm, P. Raisch und K. Tiedemann, 1988

Festschrift für Henning Piper, hrsg von W. Erdmann, W. Gloy, R. Herber, 1996 Lohn der Leistung und Rechtssicherheit, Festschrift für Albert Preu zum 70. Geburtstag, hrsg von M. Bohlig, M. Brandi-Dohrn, L. Donle, M. Buddeberg, St. Schweyer, 1988

Festschrift für Karlheinz Quack zum 65. Geburtstag am 3. Januar 1991, hrsg von H.P. Westermann und W. Rosener, 1991

Patent Practice in Japan and Europe, Liber Amicorum for Guntram Rahn, hrsg von B. Hansen und D. Schüssler-Langeheine, 2011 
FS P. Raue (2006)

FS Th. Reimann (2009)

FS W. Ritter (1997)

FS R. Rogge (2001)

FS F. J. Säcker (2006)

FS H. Salger (1995)

FS O. Sandrock (2000)

FS T. Schilling (2007)

FS H. Schippel (1996)

FS P. Schlechtriem (2003)

FS W. Schluep (1988)

FS F. Schönherr (1986)

FS G. Schricker (2005)

FS G. Schulze (2017)

FS R. Schweizer (2003)

FS I. Schwenzer (2011)

FS A. Söllner (1990)

FS A. Söllner (2000)

FS A. Sölter (1982)

FS W. Sonn (2014)

Festgabe K. Spätgens FS T. Spengler (2007)

FS D. Stauder

FS D. Stauder (2011)

Mélanges D. Stauder

FS E. Steindorff (1990)

FS R. Strasser (1983)

FS J. Straus (2009)
Festschrift für Peter Raue, hrsg von R. Jacobs, H.-J. Papier und P.-K. Schuster, 2006

Patentrecht, Festschrift für Thomas Reimann, hrsg von Ch. Osterrieth,

M. Köhler und K. Haft, 2009

Festschrift für Wolfgang Ritter zum 70. Geburtstag, hrsg von M.D. Kley ua, 1997

$=$ GRUR 2001, $865 \mathrm{ff}$

Festschrift für Franz Jürgen Säcker zum 70. Geburtstag, hrsg von D. Joost, H. Oetker M. Paschke, 2006

Strafrecht, Strafverfahrensrecht, Recht, FS für H. Salger zum Abschied aus dem Amt als Vizepräsident des Bundesgerichtshofes, hrsg von A. Eser, H.-J. Kullmann, L. Meyer-Goßner, W. Odersky, R. Voss, 1995

Festschrift für Otto Sandrock zum 70. Geburtstag, hrsg von K. P. Berger, W. Ebke, S. Elsing, B. Großfeld, G. Kühne, 2000

Festschrift für Tilman Schilling zum 70. Geburtstag am 29. Juli 2007, hrsg von M. Grosch und E. Ullmann, 2007

Festschrift für Helmut Schippel zum 65. Geburtstag, hrsg von der Bundesnotarkammer, 1996

Festschrift für Peter Schlechtriem zum 70. Geburtstag, hrsg von I. Schwenzer und G. Haager, 2003

Innominatverträge, Festgabe zum 60. Geburtstag von Walter R. Schluep, hrsg von P. Forstmoser, P. Tercier, R. Zäch, 1988

Wirtschaftsrecht in Theorie und Praxis, Gedenkschrift für F. Schönherr, hrsg von W. Barfuß, H. Torggler, Ch. Hauer, L. Wiltschek, G. Kucsko, 1986 Perspektiven des Geistigen Eigentums und Wettbewerbsrechts, Festschrift für Gerhard Schricker zum 70. Geburtstag, hrsg von A. Ohly, Th. Dreier, H.-P. Götting, M. Haedicke, M. Lehmann, 2005

Anwalt des Urheberrechts, Festschrift für Gernot Schulze zum 70. Geburtstag, hsg von Th. Dreier, K.-N. Peifer, L. Specht, 2017

Selbstbestimmung und Recht, Festgabe für Rainer J. Schweizer zum

60. Geburtstag, hrsg von P. Sutter, 2003

Private Law, national - global - comparative, Festschrift für Ingeborg

Schwenzer zum 60. Geburtstag, hrsg von A. Büchler und M. Müller-Chen, 2011

Geschichtliche Rechtswissenschaft. Freundesgabe für Alfred Söllner zum 60. Geburtstag am 5.2.1990, hrsg von G. Köbler, M. Heinze, J. Schapp, 1990

Europas universale rechtsordnungspolitische Aufgabe im Recht des dritten Jahrtausends, Festschrift für Alfred Söllner zum 70. Geburtstag, hrsg von G. Köbler ua, 2000

Wettbewerbsordnung und Wettbewerbsrealität, Festschrift für Arno Sölter zum 70. Geburtstag, hrsg von C.-A. Andreae und W. Benisch, 1982

Festschrift für Walter Sonn, hrsg von R. Beetz, D. Alge, G. Heger, P. Pawloy und A. Weinzinger, 2014

$=$ WRP 1999, $1 \mathrm{ff}$

Festschrift für Tilman Spengler, 2007

$=$ IIC 31 (2000), $625 \mathrm{ff}$

Nourriture de l'esprit, Festschrift für Dieter Stauder zum 70. Geburtstag, hrsg von H.-P. Götting und C. Schlüter, 2011

La défense des droits de la propriété industrielle en Europe, aux Etats-Unis et au Japon, Mélanges offerts à Dieter Stauder, Collection C.E.I.P.I., 2001

Festschrift für Ernst Steindorff zum 70. Geburtstag am 13. März 1990, hrsg von J.F. Baur, K.J. Hopt, K.P. Mailänder, 1990

Möglichkeiten und Grenzen der Rechtsordnung, Festschrift für Rudolf Strasser, hrsg von W. Schwarz, 1983

Patents and Technological Progress in a Globalized World - Liber Amicorum Joseph Straus, hrsg von W. Prinz zu Waldeck, W. Adelman, R. Brauneis, J. Drexl, R. Nack, 2009 
FS P. Ströbele (2019)

Festschrift für Paul Ströbele zum 75. Geburtstag, hrsg von F. Hacker und F. Thiering, 2019

FS R. Teschemacher (2008) = GRUR Int 2008, 631ff

FS W. Tilmann (2003) Festschrift für Winfried Tilmann zum 65. Geburtstag, hrsg von E. Keller, C. Plassmann und A. von Falck, 2003

FS K. Tolksdorf (2014) Festschrift für Klaus Tolksdorf, hrsg von F. Dencker, Gr. Galke und A. Voßkuhle, 2014

FS F. Traub (1994) Festschrift für Fritz Traub zum 65. Geburtstag, hrsg von C.D. Asendorf, J. Dembowski, J. Maruhn und H. Weiss, 1994

FS E. Ullmann (2006) Festschrift für Eike Ullmann, hrsg von H.-J. Ahrens, J. Bornkamm und H.P. Kunz-Hallstein, 2006

FS R. Vieregge (1995) Festschrift für Ralf Vieregge zum 70. Geburtstag am 6. November 1995, hrsg von J. Baur, R. Jacobs, M. Lieb, P.-C. Müller-Graff, 1995

FS F. Vischer (1983)

FS M. Vogel (2017)

FS R. Volhard (1996) Festschrift für Frank Vischer zum 60. Geburtstag, hrsg von P. Böckli, H. Hinderling, H.P. Tschudi, K. Eichenberger, 1983

Kreativität und Charakter, Festschrift für Martin Vogel zum 70. Geburtstag, hrsg von A. G. von Olenhusen und Th. Gergen, 2017

Recht, Geist und Kunst, liber amicorum für Rüdiger Volhard, hrsg von K. Reichert ua, 1996

FS E. von Caemmerer (1978) Festschrift für Ernst von Caemmerer zum 70. Geburtstag, hrsg von H.C. Fischer, 1978

FS H. von der Groeben (1987)

FS O.-F. von Gamm (1990)

Eine Ordnungspolitik für Europa, Festschrift für Hans von der Groeben zu seinem 80. Geburtstag, hrsg von E.-J. Mestmäcker, H. Möller und H.-P. Schwarz, 1987

FS W. von Meibom (2010) Festschrift für Otto-Friedrich Frhr. v. Gamm, hrsg von H. Erdmann, H.-K. Mees, H. Piper, O. Teplitzky, W. Hefermehl, P. Ulmer, 1990

FS A.-A. Wandtke (2013) Festschrift für Wolfgang von Meibom, hrsg von Ch. Harmsen, O.J. Jüngst und F. Rödiger, 2010

FS G. Weißmann (2003) Festschrift für Artur-Axel Wandtke zum 70. Geburtstag, hrsg von K.-I. Wöhrn, W. Bullinger, E. Grunert und C. Ohst, 2013

FS L. Wiltschek (2008)

FS L. Wiltschek (2018) Freiheit, Sicherheit, Recht. Notariat und Gesellschaft. Festschrift Georg Weißmann, hrsg von der Österr. Notarkammer (2003)

FS R. Zäch (1999) $=$ ÖBl 2008, $161 \mathrm{ff}$ $=$ ÖBl 2018, 145

Der Einfluß des europäischen Rechts auf die Schweiz, Festschrift für Prof. R. Zäch zum 60. Geburtstag, hrsg von Forstmoser, von der Crone, Weber und Zorbel, 1999

FS 10 Jahre DVS (1999) Ehrenamtliche Richter - Demokratie oder Dekoration am Richtertisch? Festschrift zum zehnjährigen Bestehen der DVS [Deutsche Vereinigung der Schöffinnen und Schöffen], hrsg von H. Lieber und U. Sens, 1999

FS 10 Jahre Rspr. GBK EPA (1996)

FS 10 Jahre sic! FS 10 Jahre Studiengang „International Studies Property Law“

Zehn Jahre Rechtsprechung der Großen Beschwerdekammer im Europäischen Patentamt, hrsg von Mitgliedern der Großen Beschwerdekammer im EPA, 1996 Das Immaterialgüterrecht und seine Schnittstellen, Sonderheft 2007

FS 25 Jahre BPatG (1986) FS 40 Jahre BPatG (2001) FS 50 Jahre BGH (2000)

Überproduktion durch Geistiges Eigentum? Festschrift zum zehnjährigen Jubiläum des Studiengangs „International Studies in Intellectual in Intellectual Property Law“, hrsg v. H.-P. Götting und A. Lunze, 2009

25 Jahre Bundespatentgericht, hrsg vom Bundespatentgericht, 1986 $=$ GRUR 2001, $545 \mathrm{ff}$

Festschrift aus Anlaß des fünfzigjährigen Bestehens von Bundesgerichtshof, Bundesanwaltschaft und Rechtsanwaltschaft beim Bundesgerichtshof, hrsg von K. Geiß, K. Nehm, E.-E. Brandner, H. Hagen, 2000 
WissFS 50 Jahre BGH (2000)

FS 50 Jahre Bundesarbeitsgericht FS 50 Jahre BPatG

FS 50 Jahre DPMA

FS 50 Jahre PAO (2017)

FS 50 Jahre VPP (2005) FS 75 Jahre Pro Honore (2000)

FS 80 Jahre Patentgerichtsbarkeit in Düsseldorf FS DPA 100 Jahre Marken-Amt (1994) FS 100 Jahre AIPPI (1997) FS 100 Jahre eidg. PatG (1988)

FS 100 Jahre GRUR (1991)

FS 200 Jahre Carl Heymanns Verlag

FS 400 Jahre Universität Gießen

Liber Amicorum Manfred Hecker (2016) Schriften zum Außenwirtschaftsrecht Bd 8
50 Jahre Bundesgerichtshof, Festgabe der Wissenschaft, hrsg von C.-W. Canaris, A. Heldrich, K.J. Hopt, C. Roxin, K. Schmidt, G. Widmaier, 4 Bde, 2000

50 Jahre Bundesarbeitsgericht, hrsg von H. Oetker ua, 2004

50 Jahre Bundespatentgericht, hrsg von A. Bender, K. Schülke, V. Winterfeldt, 2011

= Festheft 50 Jahre Deutsches Patent- und Markenamt in München, GRUR 1999, $781 \mathrm{ff}$

= Festschrift 50 Jahre Patentanwaltsordnung, hrsg von der Patentanwaltskammer, 2017

Festschrift 50 Jahre VPP, hrsg von R. Einsele und E. Franke, 2005

Gute Beispiele setzen schlechte Sitten ins Unrecht, 75 Jahre Arbeit in Grauzonen der Wirtschaft, red. von O. Dobbeck, 2000

hrsg von Th. Kühnen, 2016

AIPPI und die Entwicklung des Gewerblichen Rechtsschutzes 1897-1997

Festschrift zum 100-jährigen Bestehen des eidgenössischen Patentgesetzes Gewerblicher Rechtsschutz und Urheberrecht in Deutschland, Festschrift zum hundertjährigen Bestehen der Deutschen Vereinigung für gewerblichen Rechtsschutz und Urheberrecht und ihrer Zeitschrift, hrsg durch F.-K. Beier, A. Kraft, G. Schricker, E. Wadle, 2 Bde

Recht im Wandel europäischer und deutscher Rechtspolitik, hrsg von B. Limperg, J. Bormann, A. Filges, M.-L. Graf-Schlicker, H. Prütting, 2015

Rechtswissenschaft im Wandel, Festschrift des Fachbereichs Rechtswissenschaft zum 400jährigen Gründungsjubiläum der Justus-Liebig-Universität Gießen, hrsg von W. Gropp, M. Lipp und H. Steiger, 2007

Liber Amicorum für Manfred Hecker zum 66. Geburtstag, hrsg von I. Jung und M. Ruttig (2016)

Rechtsfragen des internationalen Schutzes geistigen Eigentums, Ehlers/ Wolffgang/Pünder (Hrsg), 2002

\section{Textausgaben, Rechtsprechungsübersichten und Hilfsmittel (weitere s 8. Aufl)}

Aus der Rechtsprechung des Bundespatentgerichts im Jahre ... (jährliche Rechtsprechungsübersichten in den Jahresberichten des BPatG und in GRUR des Folgejahrs) GRUR 1978, 404 (Maue-zur Rocklage); 1979, 341 (Starck); 1980, 815; 1981, 489; 1982, 379; 1983, 267; 1984, 479 (Schwendy); 1985, 403; 1986, 565; 1987, 325 (Ströbele); 1988, 413 (Schade); 1989, 388 (Schade/Hellebrand); 1990, 483 (Hellebrand/Ströbele); 1991, 483 (Anders/ Schade/Ströbele); 1992, 347; 1993, 631; 1994, 399 (Pösentrup/Schade/Ströbele); 1995, 365; 1996, 303 (Pösentrup/Keukenschrijver/Ströbele); 1997, 487 (Anders/ Hacker); 1998, 604; 1999, 443; 2000, 257(Anders); 2001, 276; 2002, 289; 2003, 369 (Kellerer); 2004, 361; 2005, 449; 2006, 441 (Winterfeldt); 2007, 449, 537; 2008, 553; 2009, 525, 613, 641 (Winterfeldt/Engels); 2010, 465; 2011, 561; 2012, 
Bausch

Beck Texte im dtv Beck'sche Formularsammlung

Beuskens

Deutsches Patent- und

Markenamt (Hrsg)

Düwel/Gabriel/Renz/

Teufel

Eckardt/Klett (Hrsg)

Europäisches Patentamt

(Hrsg)

Gewerblicher Rechts-

schutz, Wettbewerbsrecht,

Urheberrecht ${ }^{61}$

Götting

Götting/Meyer/

Vormbrockj (Hrsg)

Haberstrumpf/Husemann

Hasselblatt

Heinemann (Hrsg)

Hoppe/Donle

Hoppe/Donle/Hülsewig

Hoppe-Jänich

Jänich/Haupt/Cantner/

Muhsfeldt (Hrsg)

Kase

Klett/Sonntag/Wilske

Mehler/Möller (Hrsg)

Meier-Beck

Mes (Hrsg)

Mes (Hrsg)

Morawek

Offenburger

Reitzle/Butenschön/

Bergmann

Rosenberger/Wündisch
551, 673 (Engels/Morawek); 2013, 433, 545 (Engels/Morawek); 2014, 409 (Engels/Morawek); 2015, 409, 513 (Kopacek/Morawek); 2016, 537 (Kopacek/ Morawek); 2017, 545 (Kopacek/Morawek); 2018, 553 (Kopacek/Morawek); 2019, 553 (Kopacek/Morawek); 2020, 471 (Kortge/Mittenberger-Huber); zuvor unter dem Titel Die Tätigkeit des Bundespatentgerichts im Jahre ...: GRUR 1974, 268; 1975, 250; 1976, 242 (Neuperth); 1977, 588 (Maue-zur Rocklage) Nichtigkeitsrechtsprechung in Patentsachen, 4 Bde, 2000/2002 und o.J. (2007)

Patent- und Designrecht ${ }^{14}, 2018$

zum Gewerblichen Rechtsschutz und Urheberrecht ${ }^{5}, 2015$

Geistiges Eigentum und Kartellrecht (Jura kompakt), 2013

Taschenbuch des Gewerblichen Rechtsschutzes, 4 Bde (Loseblatt)

EPÜ- und PCT-Tabellen ${ }^{4}, 2016$

Wettbewerbsrecht, Gewerblicher Rechtsschutz und Urheberrecht Vorschriftensammlung, 2007

Europäisches Patentübereinkommen, Textausgabe ${ }^{14}, 2010$

(Loseblatt, Beck), 2018

Gewerblicher Rechtsschutz und Urheberrecht (Prüfe dein Wissen)³, 2015

Gewerblicher Rechtsschutz und Wettbewerbsrecht ${ }^{9}, 2020$

Wettbewerbs- und Kartellrecht, Gewerblicher Rechtsschutz ${ }^{6}, 2015$

Münchener Anwaltshandbuch Gewerblicher Rechtsschutz ${ }^{4}, 2012$

Patent- und Musterrecht (Beck-Texte 5563) ${ }^{11}, 2011$

Die Rechtsprechung der Instanzgerichte zum Patent- und Gebrauchsmusterrecht seit dem Jahr 2017, GRUR-RR 2018, 393

Die Rechtsprechung der deutschen Instanzgerichte zum Patent- und

Gbrauchsmusterrecht seit dem Jahr 2018, GRUR-RR 2019, 505

Die Rechtsprechung der Instanzgerichte zum Patent- und Gebrauchsmusterrecht seit dem Jahr 2013, GRUR-RR 2013, 473

Neue Schutzrechte in der Unternehmenspraxis, 2009

Dictionary of Industrial Property, 1980 (engl/span/franz/dt)

Intellectual Property Law in Germany, 2008

Intellectual Property, A Dictionary on Legal Terms, English-Chinese/ChineseEnglish, 2015

Die Rechtsprechung des Bundesgerichtshofs zum Patent- und Gebrauchsmusterrecht/in Patentsachen im Jahr 2005, GRUR 2007, 11; 2006, GRUR 2007, 913; 2007, GRUR 2008, 1033 = IIC 2009, 434; 2008, GRUR 2009, 893; 2009, GRUR 2010, 1041; 2010, GRUR 2011, 857; 2011, GRUR 2012, 1177; 2012, GRUR 2013, 1177; 2013, GRUR 2014, 1033; 2014, GRUR 2015, 721; 2015, GRUR 2016, 865; 2016, GRUR 2017, 1065; 2017, GRUR 2018, 977; 2018, GRUR 2019, 985

Beck'sches Prozeßformularbuch ${ }^{11}, 2010$

Münchener Prozeßformularbuch Bd 54, 2014

Aktuelle Entscheidungspraxis des BPatG zum Patentrecht, VPP-Rdbr 2014, 97;

2015, 60; 2016, 46

Patent und Patentrecherche, 2014

Gesetz über Arbeitnehmererfindungen/Act on Employees' Inventions³, 2007 Verträge über Forschung und Entwicklung ${ }^{3}, 2017$

\section{XXXIV}


Starck

Teschemacher

Trimborn

Vollrath

von Uexküll

Wuttke
Gewerblicher Rechtsschutz mit Wettbewerbs- und Urheberrecht (juris Texte), Ausgabe 2004

Aktuelle Rechtsprechung der Beschwerdekammern des EPA - Notizen aus der/für die Praxis, Mitt 2009, 297; Mitt 2018, 319

Aktuelle Entwicklungen im Erfindungsrecht sowie bei Lizenzen und Know-how im Zeitraum 2004-2006, Mitt 2006, 352

Praxis der Patent- und Gebrauchsmusteranmeldung ${ }^{5}, 2002$

Wörterbuch der Patent- und Markenpraxis ${ }^{8}$, 2011 (dt/engl, engl/dt)

Aktuelles aus dem Bereich der „Patent Litigation“, Mitt 2013, 483, Mitt 2014, 452, Mitt 2016, 55, Mitt 2017, 55, Mitt 2018, 107

\section{Zeitschriften und Entscheidungssammlungen}

\begin{tabular}{|c|c|}
\hline $\mathrm{ABl} \mathrm{EG}$ & Amtsblatt der Europäischen Gemeinschaften \\
\hline $\mathrm{ABl} \mathrm{EPA}$ & Amtsblatt des Europäischen Patentamts \\
\hline $\mathrm{AcP}$ & Archiv für civilistische Praxis \\
\hline AJP & Aktuelle juristische Praxis \\
\hline Ann. propr. ind. & Annales de la propriété industrielle, artistique et littéraire \\
\hline AntBul & The Antitrust Bulletin \\
\hline AnwBl & Anwaltsblatt \\
\hline AöR & Archiv des öffentlichen Rechts \\
\hline AP & Nachschlagewerk des Bundesarbeitsgerichts (Arbeitsrechtliche Praxis) \\
\hline $\mathrm{AuR}$ & Arbeit und Recht \\
\hline BAGE & Entscheidungen des Bundesarbeitsgerichts; s auch schweiz. BAGE \\
\hline BAnz & Bundesanzeiger \\
\hline $\mathrm{BB}$ & Der Betriebsberater \\
\hline Bek AfEP & $\begin{array}{l}\text { Bekanntmachungen des Amtes für Erfindungs- und Patentwesen der } \\
\text { Deutschen Demokratischen Republik }\end{array}$ \\
\hline BFHE & Sammlung der Entscheidungen und Gutachten des Bundesfinanzhofs \\
\hline $\mathrm{BFH} / \mathrm{NV}$ & Sammlung amtlich nicht veröffentlichter Entscheidungen des BFH \\
\hline BfS & Blatt für Sortenwesen \\
\hline $\mathrm{BGBl}$ & Bundesgesetzblatt \\
\hline BGE & Entscheidungen des schweiz. Bundesgerichtes \\
\hline BGHR & Systematische Sammlung der Entscheidungen des BGH \\
\hline BGHReport & Schnelldienst zur Zivilrechtsprechung des BGH \\
\hline BGHSt & Entscheidungen des Bundesgerichtshofs in Strafsachen \\
\hline BGHZ & Entscheidungen des Bundesgerichtshofs in Zivilsachen \\
\hline BIE & $\begin{array}{l}\text { Bijblad bij De Industriële Eigendom, seit März } 2010 \text { Berichten Industriële } \\
\text { Eigendom }\end{array}$ \\
\hline BlStSozArbR & Blatt für Steuer-, Sozialversicherungs- und Arbeitsrecht \\
\hline BlPMZ & Blatt für Patent-, Muster- und Zeichenwesen \\
\hline BOPI & Bulletin officiel de la propriété industrielle \\
\hline BPatGE & Entscheidungen des Bundespatentgerichts \\
\hline BRDrs & Bundesratsdrucksache \\
\hline BStBl & Bundessteuerblatt \\
\hline BTDrs & Bundestagsdrucksache \\
\hline BVerfGE & Entscheidungen des Bundesverfassungsgerichts \\
\hline BVerwGE & Entscheidungen des Bundesverwaltungsgerichts \\
\hline CahDrEur & Cahiers de droit européen \\
\hline CIPR & CIP-Report (Universität Düsseldorf) \\
\hline CIT & Chemie-Ingenieur-Technik \\
\hline CMLR & Common Market Law Review \\
\hline CPR & Canadian Patent Reporter/Review \\
\hline $\mathrm{CR}$ & Computer und Recht \\
\hline CRi & Computer law review international, zuvor Computer und Recht internat \\
\hline
\end{tabular}


DB

DEPATIS

DEPATISnet

DJ

$\operatorname{dn} B$

DÖD

DÖV

DPINFO

DR

DRiZ

DRZ

DuD

DVBl

ecolex

EFG

EGR

EIPR

ENPR

Entsch

EPA (Generaldirektion

Beschwerde) (Hrsg)

EPA-E

epi-Inf

EPOR

EuGRZ

EuPatBl

EurLawRev

EuV

EuZW

EWS

FSR

Gareis

GazPal

GoltdArch

GRUR

GRUR Ausl

GRUR Int

GRUR Newsletter

GRURPrax

GRUR-RR

HRR

ICLQ

IDEA

IER

IIC
Der Betrieb

elektronisches Archiv des DPMA

Datenbestände von DEPATIS online

Deutsche Justiz

Der Neuerer Teil B

Der öffentliche Dienst

Die öffentliche Verwaltung

Auskunftssystem des DPMA

Deutsches Recht

Deutsche Richterzeitung

Deutsche Rechts-Zeitschrift

Datenschutz und Datensicherung

Deutsches Verwaltungsblatt

Fachzeitschrift für Wirtschaftsrecht (Österreich)

Entscheidungen der Finanzgerichte

Entscheidungssammlung Arbeitnehmererfindungsrecht (früher:

Entscheidungssammlung Gewerblicher Rechtsschutz), hrsg von Gaul/

Bartenbach, 1972ff

European Intellectual Property Review

European National Patent Reports

Landgericht Düsseldorf, Entscheidungen der 4. Zivilkammer

Rechtsprechung der Beschwerdekammern des Europäischen Patentamts, 1996

Entscheidungen der Beschwerdekammern des EPA

EPI-Information

European Patent Office Reports

Europäische Grundrechte-Zeitschrift

Europäisches Patentblatt

European Law Review

Erfindungs- und Vorschlagswesen

Europäische Zeitschrift für Wirtschaftsrecht

Europäisches Wirtschafts- und Steuerrecht

Fleet Street Reports (früher Fleet Street Patent Law Reports)

Die patentamtlichen und gerichtlichen Entscheidungen in Patent-, Muster-

und Markenschutzsachen, einschl neuer Folge ab 189619 Bde 1881 bis

1914

Gazette du Palais (Frankreich)

Goltdammers Archiv für Strafrecht

Gewerblicher Rechtsschutz und Urheberrecht

s GRUR Int

Gewerblicher Rechtsschutz und Urheberrecht Internationaler Teil, teilweise Auslands- und Internationaler Teil, seit 2020 GRUR International: Journal of European and International IP Law

GRUR Newsletter

Gewerblicher Rechtsschutz und Urheberrecht Praxis im Immaterialgüter- und

Wettbewerbsrecht

GRUR-Rechtsprechungsreport

Höchstrichterliche Rechtsprechung

The International and Comparative Law Quarterly

Idea - The Patent, Trademark, and Copyright Journal of Research and

Education (USA)

Intellectuele Eigendom en Reclamerecht (Niederlande)

International Review of Industrial Property and Copyright Law, seit 2004

International Review of Intellectual Property and Competition Law 
IndProp

Information StW

Ing.-Cons.

InstGE

IPQ

IPRax

IPRB

iur

JA

JCP

JIBL

JIPLP

JJGrAIPPI

JMBl

JPOS

JPTOS

JR

JurBüro

JurPC

JuS

JUVE

JVBl

JW

JWIP

JWorldTrL

JZ

KritV

KWI

LiechtJZ

Liedl

LM

LMK

MA

ManIntProp

MarkenR

MDR

Mitt

MMR

MR

$\mathrm{MuW}$

NedJ

NIR

NJ

NJB

NJOZ

NJW

NJW-CoR

NJW-RR

NJWE-VHR

NJWE-WettbR NordÖR
Industrial Property, seit 1995 Industrial Property and Copyright (s ProprInd)

Information über Steuer und Wirtschaft

L’Ingénieur-Conseil, Revue technique et juridique des droits intellectuels

(Belgien)

Entscheidungen der Instanzgerichte zum Recht des Geistigen Eigentums, bisher 13 Bde

Intellectual Property Quarterly (London)

Praxis des Internationalen Privat- und Verfahrensrechts

Der IP-Rechtsberater

Informatik und Recht

Juristische Arbeitsblätter

Juris-classeur périodique

Journal of International Biotechnology Law

Journal of Intellectual Property Law \& Practice

Journal of the Japanese Group of AIPPI

Justizministerialblatt

Journal of the Patent Office Society

Journal of the Patent and Trademarks Office Society

Juristische Rundschau

Das juristische Büro

Jur-PC (Zeitschrift, im Internet)

Juristische Schulung

JUVE Rechtsmarkt

Justizverwaltungsblatt

Juristische Wochenschrift

Journal of World Intellectual Property

Journal of World Trade Law

Juristenzeitung

Kritische Vierteljahresschrift für Gesetzgebung und Rechtswissenschaft

Karlsruher Schriften zum Wettbewerbs- und Immaterialgüterrecht

Liechtensteinische Juristen-Zeitung

Liedl, Entscheidungen des Bundesgerichtshofs in Zivilsachen, Patentnichtig-

keitsklagen

Lindenmaier/Möhring, Nachschlagewerk des Bundesgerichtshofs

Kommentierte BGH-Rechtsprechung Lindenmaier-Möhring

Der Markenartikel

Managing intellectual property

Markenrecht

Monatsschrift für Deutsches Recht

Mitteilungen der deutschen Patentanwälte, zeitweise Mitteilungen aus dem

Verband der deutschen Patentanwälte

Multimedia und Recht

Medien und Recht (Österreich)

Markenschutz und Wettbewerb

Nederlandse Jurisprudentie

Nordisk Immateriellt Rättsskydd (Schweden)

Neue Justiz

Nederlands Juristenblad

Neue Juristische Online-Zeitschrift

Neue juristische Wochenschrift

NJW-Computerreport

NJW-Rechtsprechungs-Report

NJW-Entscheidungsdienst Versicherungs- und Haftungsrecht

NJW-Entscheidungsdienst Wettbewerbsrecht

Zs für öffentliches Recht in Norddeutschland 
NVwZ

NVwZ-RR

NZA

NZBau

NZG

NZI

NZKart

ÖBl

OGZ

ÖJZ

OLGR

OLGZ

öPBl

PatBl

PatentiLic

PatLic

PatWorld

PharmR

PharmInd

PIBD

ProprInd

PTCJ

RdA

RDPI

RDV

Rev.tr.dr.comm.

Rev.tr.dr.eur.

RGBl

RGSt

RGZ

RiA

RIPIA

Riv.dir.ind.

Riv.propr.intell. e ind.

RIW

\section{ROHGE}

ROW

RPC

RRPI

RsprDB ArbEG

Rpfleger

RzU

Schulte-Kartei

SchwPMMBl

sic!

SJZ

Slg

SMI

StBp
Neue Zeitschrift für Verwaltungsrecht

Neue Zeitschrift für Verwaltungsrecht Rechtsprechungs-Report

Neue Zeitschrift für Arbeits- und Sozialrecht

Neue Zeitschrift für Baurecht und Vergaberecht

Neue Zeitschrift für Gesellschaftsrecht

Neue Zeitschrift für das Recht der Insolvenz und Sanierung

Neue Zeitschrift für Kartellrecht

Österreichische Blätter für gewerblichen Rechtsschutz und Urheberrecht

Entscheidungen des Obersten Gerichts der DDR in Zivilsachen

Österreichische Juristen-Zeitung

OLG-Report (mit Zusatz des Gerichts)

Entscheidungen der Oberlandesgerichte in Zivilsachen

(österreichisches) Patentblatt

Patentblatt

Patenti i Licenzii

Patent and licensing

Patent World, London

Pharma-Recht

Pharmazeutische Industrie

Propriété industrielle - Bulletin de documentation

La Propriété Industrielle, seit 1995 La Propriété Industrielle et le Droit

d'auteur, bis Mai 1998, seither Revue de l'OMPI

Patent, Trademark and Copyright Journal

Recht der Arbeit

Revue du droit de la propriété industrielle (Frankreich)

Recht der Datenverarbeitung

Revue trimestrielle du droit commerciel (Frankreich)

Revue trimestrielle de droit européen (Frankreich)

Reichsgesetzblatt

Entscheidungen des Reichsgerichts in Strafsachen

Entscheidungen des Reichsgerichts in Zivilsachen

Recht im Amt

Revue internationale de la propriété industrielle et artistique

(Frankreich)

Rivista di diritto industriale (Italien)

Rivista della proprietà intellettuale ed industriale (Italien)

Recht der Internationalen Wirtschaft, zeitweise RIW/AWD

(Außenwirtschaftsdienst des Betriebs-Beraters)

Entscheidungen des Reichsoberhandelsgerichts

Recht in Ost und West

Reports of Patent, Design and Trade mark cases

Revista romana de proprietate industriala, Bukarest

Hellebrand/Schmidt Rechtsprechungsdatenbank ArbEG ${ }^{5}, 2012$

Der deutsche Rechtspfleger

Rechtsprechung zum Urheberrecht

hrsg von Schulte (auch als CD-ROM)

Schweizerisches Patent-, Muster- und Markenblatt

(schweiz.) ZS für Immaterialgüter-, Informations- und Wettbewerbsrecht

(ab 1997, zuvor SMI)

Schweizerische Juristen-Zeitung

Sammlung der Entscheidungen des Europäischen Gerichtshofs

Schweizerische Mitteilungen über Immaterialgüterrecht (bis 1996), bis 1984

Schweizerische Mitteilungen über gewerblichen Rechtsschutz und Urheber-

recht, ab 1997 sic!

Die steuerliche Betriebsprüfung 
UFITA

USPQ

VerwArch

VPP-Rdbr

wbl

WIB

WIPR

WiRO

WissR

wistra

WM

WRP

WuW

ZAkDR

ZBR

ZEuP

ZEV

ZGE

ZfA

ZfRV

ZGE

ZGR

ZHR

ZinsO

ZIP

ZJS

ZÖR

ZRP

ZUM

ZUR

ZVglRWiss

ZWeR

ZZP

ZZP Int
Archiv für Urheber-, Film-, Funk- und Theaterrecht

The United States Patent Quarterly (USA)

Verwaltungsarchiv

VPP-Rundbrief

Wirtschaftsrechtliche Blätter

Wirtschaftsrechtliche Beratung

World Intellectual Property Report, London

Wirtschaft und Recht in Osteuropa

Wissenschaftsrecht

ZS für Wirtschaft, Steuer, Strafrecht

Wertpapier-Mitteilungen Teil IV

Wettbewerb in Recht und Praxis

Wirtschaft und Wettbewerb

Zeitschrift der Akademie für Deutsches Recht

Zeitschrift für Beamtenrecht

Zeitschrift für europäisches Privatrecht

Zeitschrift für Erbrecht und Vermögensnachfolge

Zeitschrift für Geistiges Eigentum

Zeitschrift für Arbeitsrecht

Zeitschrift für Rechtsvergleichung, int. Privatrecht und Europarecht

(Österreich)

Zeitschrift für Geistiges Eigentum

Zeitschrift für gewerblichen Rechtsschutz

Zeitschrift für das gesamte Handelsrecht und Wirtschaftsrecht

Zeitschrift für das gesamte Insolvenzrecht

Zeitschrift für Wirtschaftsrecht (und Insolvenzpraxis)

Zeitschrift für das juristische Studium

Zeitschrift für öffentliches Recht

Zeitschrift für Rechtspolitik

Zeitschrift für Urheber- und Medienrecht

Zeitschrift für Umweltrecht

Zeitschrift für vergleichende Rechtswissenschaft

Zeitschrift für Wettbewerbsrecht

Zeitschrift für Zivilprozess

Zeitschrift für Zivilprozess International 
